\title{
The beauty is in the decoration
}

Designer Dirac fermions and topological phases in molecular graphene

Kenjiro K. Gomes, Warren Mar, Wonhee Ko, Francisco Guinea, Hari C. Manoharan

Nature 483, 306 (2012)

\section{Recommended with a commentary by Felix von Oppen, Freie Universität Berlin}

Over the years, the field of graphene has proven a remarkable source of new physics, often related to its massless Dirac-like bandstructure. In addition, graphene inspired deep insights into the phases of matter. It was in the context of graphene that the quantum spin-Hall effect was first thought of [1], an insight which flourished into the field now known as topological insulators. As it turns out, the spin-orbit interaction is quite weak in graphene so that its spin-Hall effect remains inaccessible to experiment. This provides but one motivation for pursuing alternative graphene-like systems which promise access to new parameter regimes.

An elegant approach to realize artificial graphene was recently taken by H.C. Manoharan and collaborators. Their experiment exploits that scanning tunneling microscopes allow for manipulating atoms or molecules adsorbed on metallic surfaces with atomic precision. This is used to arrange $\mathrm{CO}$ molecules into a hexagonal lattice on a $\mathrm{Cu}$ (111) surface which is known to have a band of surface states. The $\mathrm{CO}$ molecules impose a periodic potential on the surface electrons, resulting in a graphene-like bandstructure. When the lattice spacing is chosen on a nanometer scale, comparable to the Fermi wavelength of the surface electrons, this artificial-graphene system has its Fermi energy close to the Dirac point.

The lattices created in this experiment, consisting of hundreds of $\mathrm{CO}$ molecules, could then be probed by scanning tunneling spectroscopy. The differential tunneling conductance provides a good measure of the local density of states as a function of energy. By measuring the tunneling conductance near the center of such a lattice, the authors confirmed the approximately linear dependence of the density of states on energy expected for a Dirac cone. The local nature of scanning tunneling spectroscopy allows for probing the two ( $A$ and $B$ ) sublattices separately and hence to address the pseudospin degree of freedom of the corresponding Dirac equation. The authors exploited this to extract the massless Dirac spectrum directly from experiment.

Even with the constraints of the registry with the underlying $\mathrm{Cu}$ surface, the $\mathrm{CO}$ lattice can be realized with different lattice spacings. This was used to realize pn-junctions which were sharp on the scale of one lattice spacing. One way to think about the shift of the Dirac point with lattice spacing is as a consequence of the familiar (scalar) deformation potential associated with a lattice distortion. More general lattice distortions generate not only scalar but also vector potentials in the Dirac equation. The authors explored these as well by creating local defects in the honeycomb 
lattice, both pseudospin conserving and pseudospin breaking, and measuring the distinct differences in the rotational symmetry of the local density of states around the defect.

Perhaps the most striking illustration of the opportunities afforded by artificial graphene is provided by two large-scale lattice distortions. In one experiment, the authors added additional $\mathrm{CO}$ molecules within the hexagons in a 'Mercedes' arrangement. Within a tight-binding picture, this modulates the hopping amplitudes between neighboring sites in an alternating fashion, known as a Kekule pattern. This endows the Dirac fermions with a mass, and indeed the authors observe that the local density of states now develops a gap. In another experiment, the $\mathrm{CO}$ molecules form a hexagonal lattice distorted in such a way that the strain-induced vector potential corresponds to a uniform magnetic field acting on the Dirac fermions (albeit with opposite signs in the two valleys to preserve time reversal symmetry). The magnetic field with strengths reaching 60 Tesla causes the formation of a zeroth Landau level, a hallmark of Dirac physics. The sublattice asymmetry of the zeroth Landau level leads to the development of a zero-bias peak when measuring the differential tunneling conductance on one sublattice and to the development of a Landau gap when measuring on the other.

This experiment complements a number of approaches to realize artificial graphene (see Ref. [2] for a recent review). The major driving force behind this research is the flexibility to tune parameters in these systems which promises access to correlated or topological phases which are inaccessible in natural graphene. The setting of the present experiment may not be particularly conducive for the realization of manybody effects since the electron-electron interaction is strongly screened by the bulk electrons of the $\mathrm{Cu}$ substrate. Prospects for the realization of topological phases could be considerably more favorable, e.g., by employing substrates with stronger spin-orbit coupling.

[1] C. L. Kane and E. J. Mele, Phys. Rev. Lett. 95, 226801 (2005)

[2] M. Polini, F. Guinea, M. Lewenstein, H. C. Manoharan, V. Pellegrini , Artificial graphene as a tunable Dirac material, arXiv:1304.0750. 